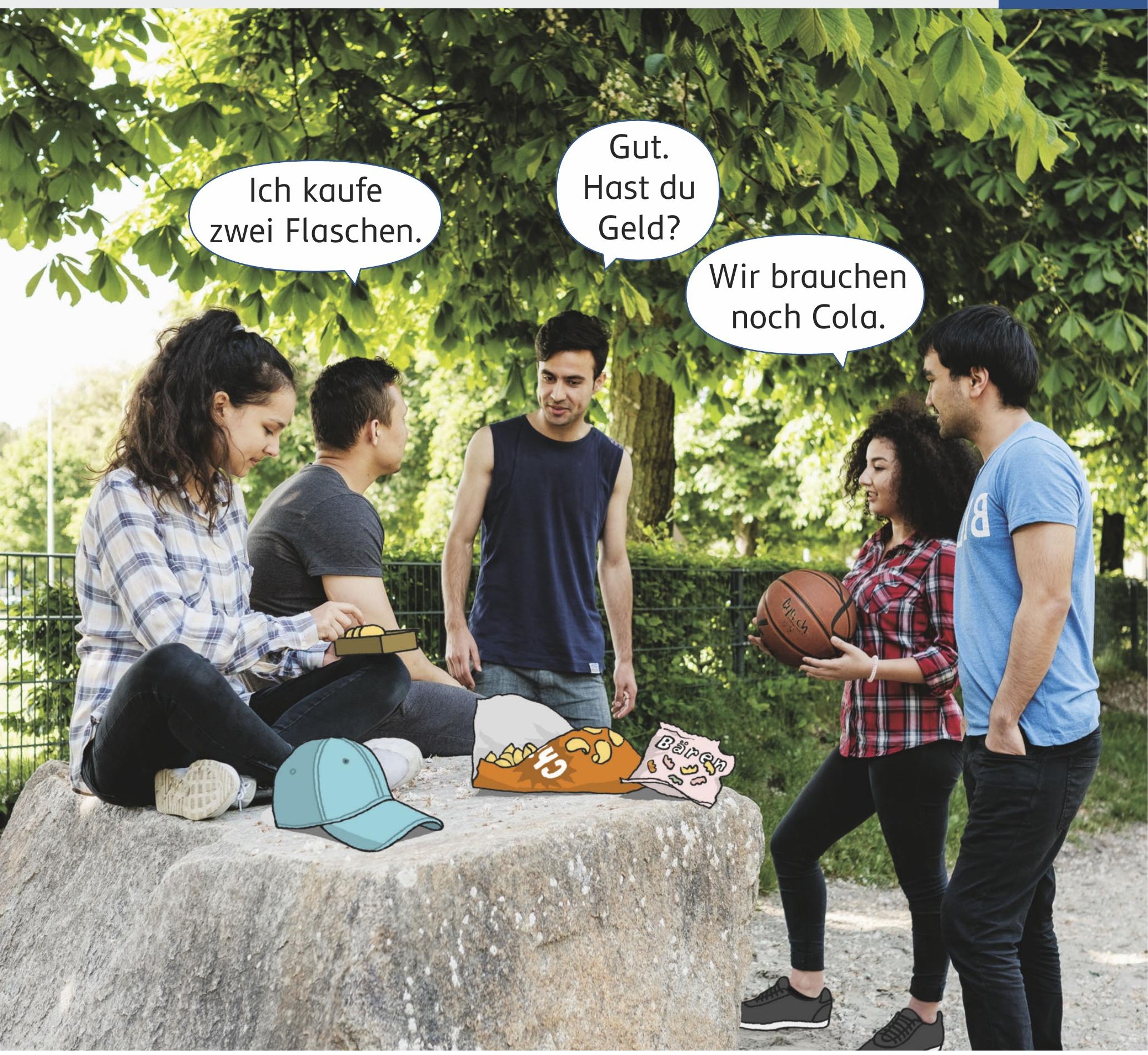

1 Was siehst du? Sprich.

2 Höre und sprich nach. (2) (9)

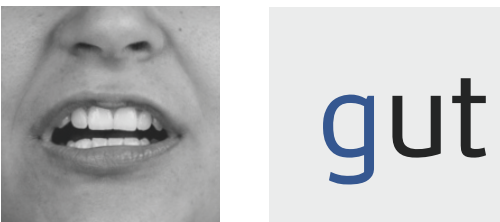




\section{Im Garten}

3a Sprich nach und fahre mit dem Finger nach. (2) (1)
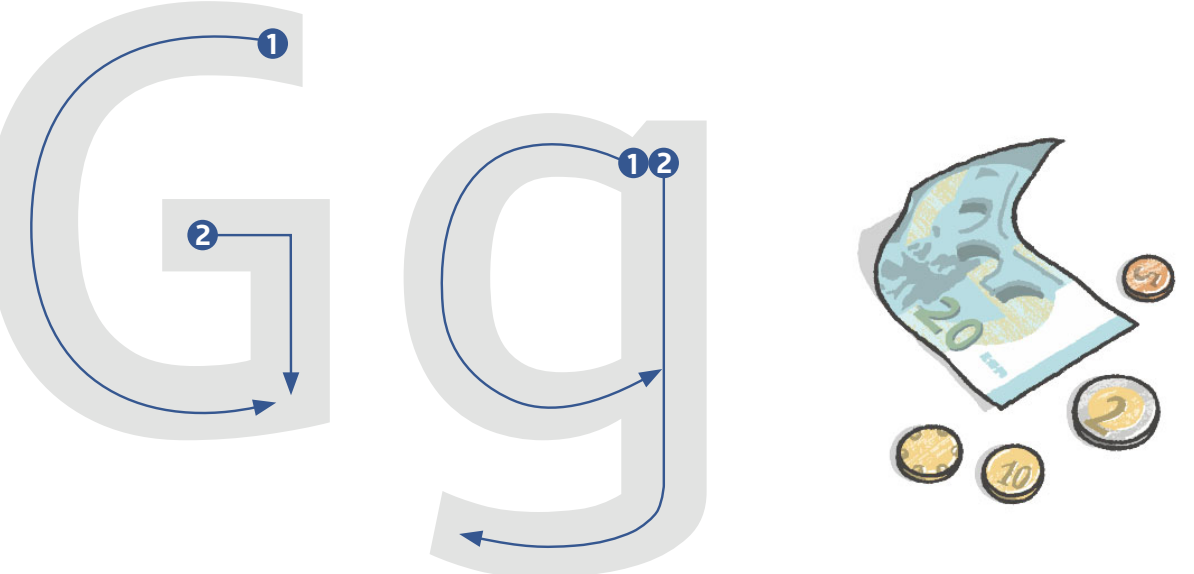

3b Schreibe.

\section{G}

g

\section{$\mathrm{Gg}$}

das Geld

3c Schreibe G g oder das ganze Wort nach.

das Geld

der Garten

grün

gelb groß

das Regal

das Glas

das Geschirr die Gabel

gern

gut

gehen sagen

das Gras

das Getränk

die Gurke

4 In welchem Wort hörst du G g? Kreuze an. (2) (1)
$\mathbf{0} \square$
$2 \square$
3 $\square$
( $\square$
(5)
- $\square$ 
$5 a$ Lies und male. 目

Das Haus hat vier Fenster.

Es hat eine Tür.

Im Garten steht ein Baum.

Im Garten stehen ein Tisch

und zwei Stühle.

5b Was ist im Garten? Kreuze an.
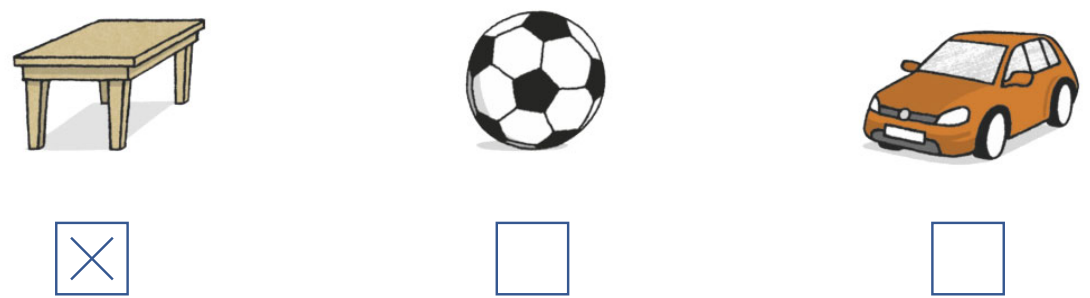

$\square$

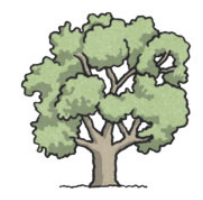

$\square$

5c Lies und schreibe Sätze.
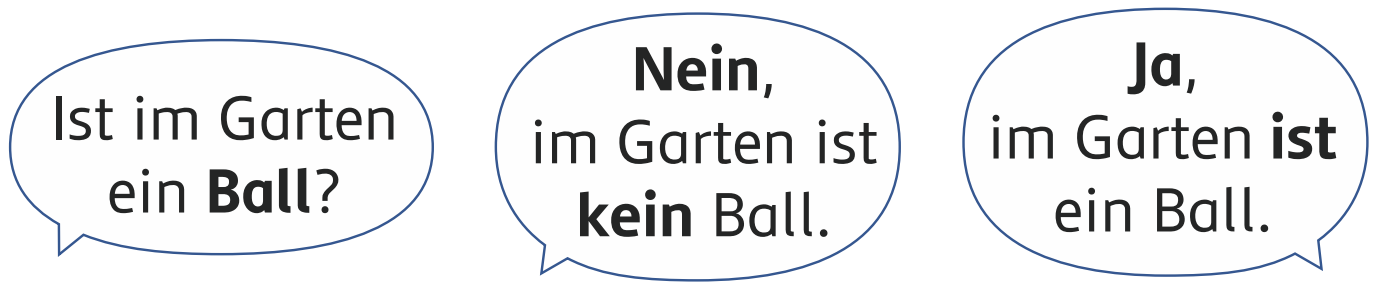

$$
\begin{aligned}
& \text { ein } \rightarrow \text { kein } \\
& \text { eine } \rightarrow \text { keine } \\
& \text { ein } \rightarrow \text { kein }
\end{aligned}
$$




\section{Im Garten}

6a Lies und markiere die Verben. 目

Ich bin im Garten. Ali ist auch im Garten. Wir sind zusammen hier. Laura ist im Kaufhaus. Das Kind ist in der Schule. Morgen sind Laura und Ali im Café. Und wo bist du? Wo seid ihr?

6b Schreibe.

\begin{tabular}{|l|l|l|l|}
\hline \multicolumn{3}{|c|}{ sein } \\
\hline ich & & wir & \\
\hline du & & ihr & \\
\hline er/sie/es & & sie/Sie & \\
\hline
\end{tabular}

7 Lies und schreibe die Anwort. 国

Die Klasse möchte grillen. Was brauchen sie?

Wer hat Messer, Gabeln und Löffel? Wer hat Teller und Gläser?

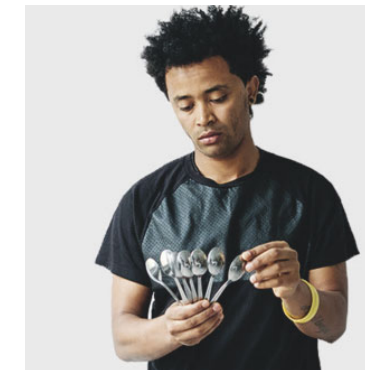

Mickel

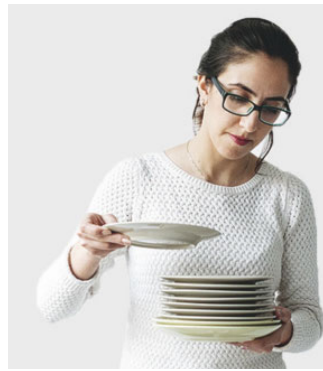

Fatima

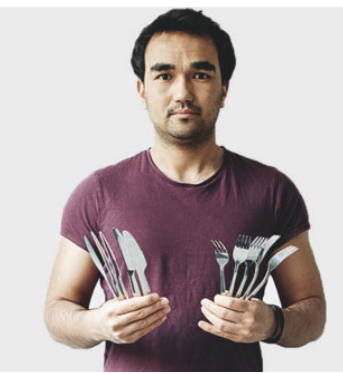

Carlos

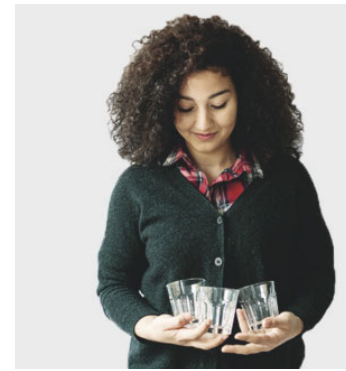

Sana

Mickel hat sieben 
8a Was brauchen die Personen? Höre und schreibe. (2) (1)

(

2

(3)

(4)

8b Sprecht zusammen.

Was brauchst du?

Ich brauche ein Heft.

9a Schreibe die Wörter.

das Messer das Glas die Gabel haben brauchen das Getränk essen

trinken

\begin{tabular}{|l|l|}
\hline Nomen & \\
\hline & \\
\hline & \\
\hline & \\
\hline & \\
\hline
\end{tabular}

9b Schreibe Sätze.

Ich brauche 


\section{Im Garten}

10 Schreibe.

But Das ist kein Tisch. B Das ist_Bus.

Das ist ein Schrank. \& Das ist_Löffel.

(5) Das ist_ Sofa. Das ist_ Messer.

$\begin{array}{lll}\text { Das ist } & \text { Getränk. } & \text { Buch. } \\ \text { Das ist } & \text { Gurke. } & \text { Tast } \\ \text { Gabel. }\end{array}$

11 Trenne die Wörter und schreibe die Sätze.

ICH|BRAUCHEEINMESSERUNDEINEGABEL.

Ich

DUKAUFSTZWEIGURKENUNDKEINEWURST.

FRAUAZIZIHATEINHANDY.ESISTNEU.

TRINKTHERRMÜLLERDENTEEMITHONIG? 
12a Höre und lies mit. (2) (1) 27 영

Ich heiße Musa. Ich bin 17 Jahre alt.

Wie heißt du?

Wie alt bist du?

Und woher

kommst du?

12b Meine Aufgabe für dich. Schreibe den Text richtig.

ich bin im garten ali ist auch im garten. Wir sind zusammen hier. Laura ist im kauthaus. das kind ist in der schule. morgen sind Laura und ali im café. und wo bist du? wo seid du?

12c Schreibe eine eigene Aufgabe. 
$\star$ Was ist kein Getränk? Streiche durch.
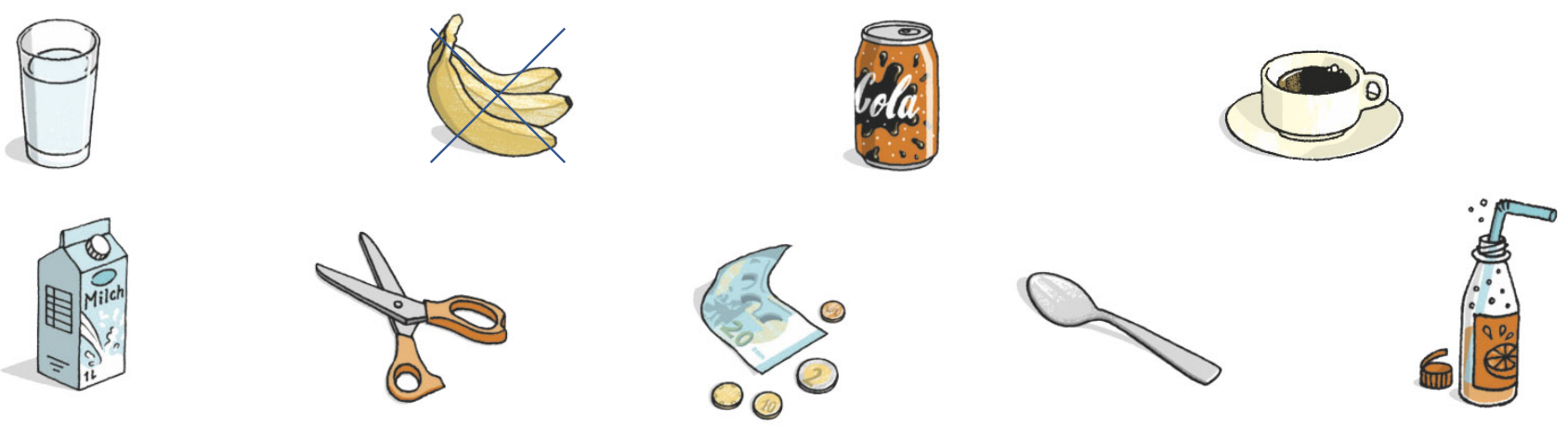

$\star \star$ Schreibe.

Musa

17 Jahre alt.

das ein Stuhl? Nein, das

zwei Tische.

$\star \star \star$ Ein oder kein? Schreibe und streiche durch.

Ist das ein Elefant?

Nein , das ist ein/kein Elefant. Das ist ein Löwe.

Ist das ein Haus?

, das ist ein / kein Haus. Das ist ein Auto.

Ist das ein Glas?

, das ist ein/kein Glas. Das ist ein Regal.

Ist das eine Frau?

, das ist eine/keine Frau. Sie heißt Sarah. 
$\star$ Verbinde Silben und Bilder. Schreibe.

$\begin{array}{lll}\text { der } & \text { Mes fel das } \\ \text { das } & \text { Ga } & \text { ser } \\ \text { die } & \text { Löf } & \text { bel }\end{array}$

$\star \star$ Schreibe.

das Mes

$r$

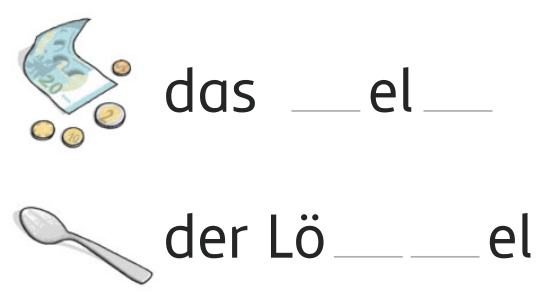

die Gab

$\star \star \star$ Schreibe die Antwort.

Was hast du?

Ich habe ein Handy.

Was brauchst du?

Was kaufst du?

Was möchtest du? 
Schreibe.

das Geld

die Gabel, die Gabeln

das Messer, die Messer

der Löffel, die Löffel

gut

Lies und sprich.

Ist im Garten

Hast du

ein Handy?

ein Tisch?

Ja, ich habe ein Handy.

Ist im Garten ein Ball?

Ja, im Garten ist ein Tisch.

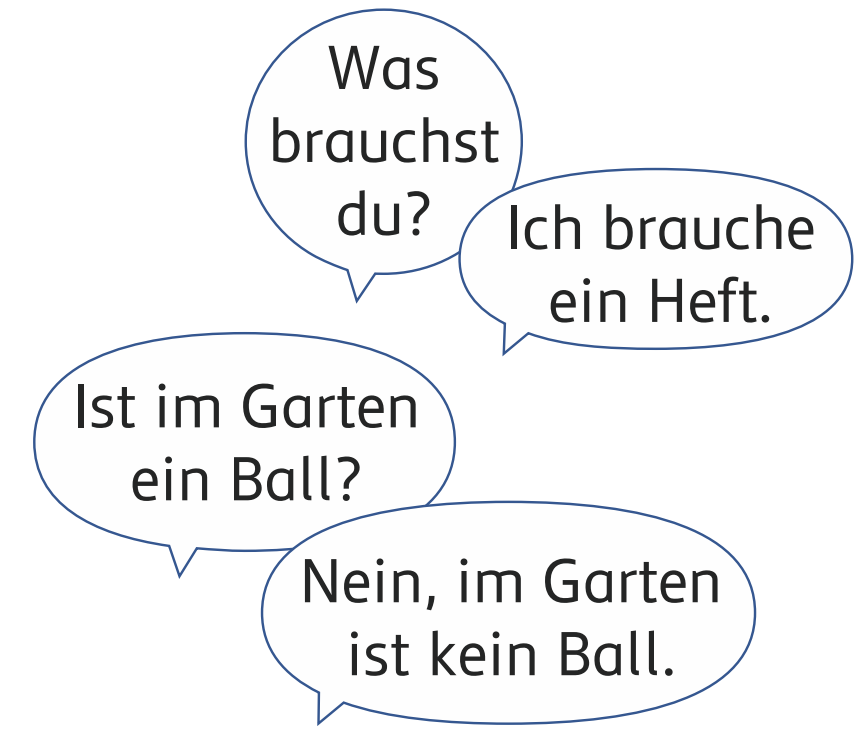




\section{Lernen unterwegs}

Mein Garten. Male und schreibe einen Text.

Im Garten steht ein Baum. 
Open Access Dieses Kapitel wird unter der Creative Commons Namensnennung - Nicht kommerziell - Keine Bearbeitung 4.0 International Lizenz (http://creativecommons.org/licenses/by-nc-nd/4.0/deed.de) veröffentlicht, welche die nicht-kommerzielle Nutzung, Vervielfältigung, Verbreitung und Wiedergabe in jeglichem Medium und Format erlaubt, sofern Sie den/die ursprünglichen Autor(en) und die Quelle ordnungsgemäß nennen, einen Link zur Creative Commons Lizenz beifügen und angeben, ob Änderungen vorgenommen wurden. Die Lizenz gibt Ihnen nicht das Recht, bearbeitete oder sonst wie umgestaltete Fassungen dieses Werkes zu verbreiten oder öffentlich wiederzugeben.

Die in diesem Kapitel enthaltenen Bilder und sonstiges Drittmaterial unterliegen ebenfalls der genannten Creative Commons Lizenz, sofern sich aus der Abbildungslegende nichts anderes ergibt. Sofern das betreffende Material nicht unter der genannten Creative Commons Lizenz steht und die betreffende Handlung nicht nach gesetzlichen Vorschriften erlaubt ist, ist auch für die oben aufgeführten nicht-kommerziellen Weiterverwendungen des Materials die Einwilligung des jeweiligen Rechteinhabers einzuholen. 